\title{
An Exceptionally Mild Catalytic Thioester Aldol Reaction Inspired By Polyketide Biosynthesis
}

\author{
Gojko Lalic, Allen D. Aloise, and Matthew D. Shair* \\ Department of Chemistry and Chemical Biology \\ Harvard University \\ Cambridge, Massachusetts 02138
}

\section{Supporting Information}

\section{Experimental Section}

General procedures: Unless otherwise noted, reactions were performed in oven dry glassware, under an atmosphere of argon. Flash column chromatography was performed using a HPFC Biotage system (Biotage Inc.) with pre-packed FLASH silica gel columns.

Materials: Unless otherwise noted solvents used were purified by passage through two packed columns of neutral alumina on a solvent dispensing system. Commercially available compounds were used without purification. TLC analyses were performed on $250 \mu \mathrm{m}$ Silica Gel $60 \mathrm{~F}_{254}$ plates purchased from EM Science.

Instruments: IR spectra were recorded on a Perkin Elmer Spectrum One FT-IR spectrometer. ${ }^{1} \mathrm{H}$ and ${ }^{13} \mathrm{C}$ NMR spectra were recorded on a Varian INOVA500 or Mercury400 spectrometer. Chemical shifts for proton and carbon resonances are reported in ppm $(\delta)$ relative to chloroform ( $\delta 7.26,77.07$ respectively). HPLC analyses were performed on a HP 1100 series instrument using the indicated column.

General procedure for the preparation of malonic acid half thioesters (2) and (3): ${ }^{1}$ To a solution of ethyl polyphosphate (PPE) $(15 \mathrm{~g})$ in $\mathrm{CHCl}_{3}(60 \mathrm{~mL})$ and THF $(10 \mathrm{~mL})$ was added malonic or methylmalonic acid (100 mmol, 4 equiv). To the resulting white suspension was added the benzyl mercaptan $\left(25 \mathrm{mmol}, 1\right.$ equiv) via syringe. The reaction was stirred at $25^{\circ} \mathrm{C}$ for $40 \mathrm{hr}$, diluted with $\mathrm{Et}_{2} \mathrm{O}(200 \mathrm{~mL})$, and extracted with saturated aq. $\mathrm{NaHCO}_{3}(3 \mathrm{X})$. The aqueous extracts were acidified with $10 \% \mathrm{HCl}$ to a $\mathrm{pH}=1-2$ and then extracted with $\mathrm{CHCl}_{3}(2 \mathrm{X})$. The organic layers were concentrated to a yellow oil and then purified via silica gel chromatography (1:1 EtOAc/hexane) to afford a yellow oil which solidified to a white solid upon standing.

General procedure for the catalytic acetate aldol reaction (Table 1): Aldol reactions were performed in capped glass vials that were not treated or dried prior to the experiments. No measures were taken to protect the reactions from the air. To a stirred solution of malonic acid half benzylthioester $(0.5 \mathrm{mmol}, 1.0$ equiv) in THF $(5 \mathrm{~mL}$, solvent stored in a vial without protection from the air before use) at $23^{\circ} \mathrm{C}$ was added 5 -methoxybenzimidazole $(0.11 \mathrm{mmol}, 0.22$ equiv) and $\mathrm{Cu}(2-$ ethylhexanoate $)_{2}(0.1 \mathrm{mmol}, 0.2$ equiv $)$. After the reaction became homogenous $(\sim 1 \mathrm{~min})$ aldehyde 
( 0.5 mmol, 1.0 equiv) was added. The solution was stirred at $23^{\circ} \mathrm{C}$ for the period of time indicated in Table 1 and quenched with a $0.5 \mathrm{M} \mathrm{HCl}$ solution. The resulting solution was diluted with EtOAc and washed successively with $0.5 \mathrm{M} \mathrm{HCl}$, saturated aq. $\mathrm{NaHCO}_{3}$, and brine. The organic layer was dried over oven dried $\mathrm{Na}_{2} \mathrm{SO}_{4}$, filtered through cotton, and concentrated under reduced pressure. The product was purified using continuous gradient flash column chromatography.

\section{Table 1, Entry 1}

3-Hydroxy-5-phenyl-pentanethioic acid S-benzyl ester: Obtained as a colorless oil in 82\% yield. FTIR (neat, $\mathrm{cm}^{-1}$ ) 3437 (b), 3027, 2926, 1683, 1494, 1451, 1071; ${ }^{1} \mathrm{H}$ NMR $\left(500 \mathrm{MHz}, \mathrm{CDCl}_{3}\right) \delta$ 7.29-7.15 (m, 10H), $4.11(\mathrm{~s}, 2 \mathrm{H}), 4.10-4.04(\mathrm{~m}, 1 \mathrm{H}), 2.90-2.60(\mathrm{~m}, 5 \mathrm{H}), 1.85-1.68(\mathrm{~m}, 2 \mathrm{H}) ;{ }^{13} \mathrm{C}$ NMR $\left(100 \mathrm{MHz}, \mathrm{CDCl}_{3}\right) \delta 198.7,141.5,137.2,128.8,128.7,128.5,127.4,126.0,67.9,50.4,38.1$, 33.3, 31.7; MS $\left(\mathrm{APCI}^{+}\right)$calcd for $\mathrm{C}_{18} \mathrm{H}_{21} \mathrm{O}_{2} \mathrm{~S}(\mathrm{M}+\mathrm{H})^{+}$: 301.1 , found 301.0.

\section{Table 1, Entry 2}

3-Hydroxy-4-methyl-pentanethioic acid S-benzyl ester: Obtained as a colorless oil in 85\% yield. FTIR (neat, $\mathrm{cm}^{-1}$ ) 3457 (b), 3063, 3030, 2961, 2874, 1679, 1051, 997; $\left.{ }^{1} \mathrm{H} \mathrm{NMR} \mathrm{(500} \mathrm{MHz,} \mathrm{CDCl}_{3}\right) \delta$ 7.32-7.25 (m, 5H), 4.18-4.12 (m, 2H), 3.88-3.84 (m, 1H), $2.75\left(\mathrm{dd}, J_{1}=15.5 \mathrm{~Hz}, J_{2}=3 \mathrm{~Hz}, 1 \mathrm{H}\right), 2.68$ $\left(\mathrm{dd}, J_{1}=15.5 \mathrm{~Hz}, J_{2}=9 \mathrm{~Hz}, 1 \mathrm{H}\right), 1.75-1.69(\mathrm{~m}, 1 \mathrm{H}), 0.95-0.92(\mathrm{~m}, 6 \mathrm{H}) ;{ }^{13} \mathrm{C} \mathrm{NMR}(100 \mathrm{MHz}$, $\left.\mathrm{CDCl}_{3}\right) \delta 199.1,137.2,128.8,128.7,127.3,73.2,47.6,33.3,33.2,18.3,17.5 ; \mathrm{MS}\left(\mathrm{APCI}^{+}\right)$calcd for $\mathrm{C}_{13} \mathrm{H}_{19} \mathrm{O}_{2} \mathrm{~S}(\mathrm{M}+\mathrm{H})^{+}:$239.1, found 239.1.

\section{Table 1, Entry 3}

3-Benzylsulfanylcarbonyl-2-hydroxy-propionic acid ethyl ester: Obtained as a colorless oil in 97\% yield. FTIR (neat, $\mathrm{cm}^{-1}$ ) 3478 (b), 3030, 2982, 2930, 1733, 1684, 1496, 1454, 1203, 1098, 1044; ${ }^{1} \mathrm{H}$ NMR $\left(500 \mathrm{MHz}, \mathrm{CDCl}_{3}\right) \delta 7.25-7.20(\mathrm{~m}, 5 \mathrm{H}), 4.49\left(\mathrm{dd}, J_{l}=6.5 \mathrm{~Hz}, J_{2}=4 \mathrm{~Hz}, 1 \mathrm{H}\right), 4.23-$ $4.18(\mathrm{~m}, 2 \mathrm{H}), 4.12-4.11(\mathrm{~m}, 2 \mathrm{H}), 3.06\left(\mathrm{dd}, J_{1}=16 \mathrm{~Hz}, J_{2}=4.5 \mathrm{~Hz}, 1 \mathrm{H}\right), 2.97\left(\mathrm{dd}, J_{1}=16 \mathrm{~Hz}, J_{2}=\right.$ $6.5 \mathrm{~Hz}, 1 \mathrm{H}), 1.23\left(\mathrm{t}, J_{1}=7.5 \mathrm{~Hz}, 3 \mathrm{H}\right) ;{ }^{13} \mathrm{C} \mathrm{NMR}\left(100 \mathrm{MHz}, \mathrm{CDCl}_{3}\right) \delta 195.6,173.2,137.0,128.8$, $128.6,127.3,67.3,62.1,47.1,33.3,14.0$

\section{Table 1, Entry 4}

3-Cyclohexyl-3-hydroxy-thiopropionic acid S-benzyl ester: Obtained as a colorless oil in 81\% yield. FTIR (neat, $\mathrm{cm}^{-1}$ ) 3462 (b), 2924, 1684, 1451, 1039; ${ }^{1} \mathrm{H}$ NMR (500 MHz, $\left.\mathrm{CDCl}_{3}\right) \delta$ 7.29-7.20 $(\mathrm{m}, 5 \mathrm{H}), 4.15-4.08(\mathrm{~m}, 2 \mathrm{H}), 3.83-3.80(\mathrm{~m}, 1 \mathrm{H}), 2.74\left(\mathrm{dd}, J_{1}=16 \mathrm{~Hz}, J_{2}=3 \mathrm{~Hz}, 1 \mathrm{H}\right), 2.67\left(\mathrm{dd}, J_{1}=\right.$ $\left.16 \mathrm{~Hz}, J_{2}=9 \mathrm{~Hz}, 1 \mathrm{H}\right), 2.56$ (bs, $\left.1 \mathrm{H}\right), 1.81-1.69(\mathrm{~m}, 3 \mathrm{H}), 1.64-1.61(\mathrm{~m}, 2 \mathrm{H}), 1.34-1.30(\mathrm{~m}, 1 \mathrm{H}), 1.22-$ $0.95(\mathrm{~m}, 5 \mathrm{H}) ;{ }^{13} \mathrm{C} \mathrm{NMR}\left(125 \mathrm{MHz}, \mathrm{CDCl}_{3}\right) \delta 199.2,137.2,128.8,128.6,127.3,72.7,47.8,43.1$, 33.2, 28.8, 28.0, 26.3, 26.1, 26.0; $\mathrm{MS}\left(\mathrm{APCI}^{+}\right)$calcd for $\mathrm{C}_{16} \mathrm{H}_{23} \mathrm{O}_{2} \mathrm{~S}(\mathrm{M}+\mathrm{H})^{+}:$279.1, found 279.0.

\section{Table 1, Entry 5}

3-Hydroxy-3-(tetrahydro-furan-3-yl)-thiopropionic acid S-benzyl ester: Obtained as a 1:1 mixture of isomers and as a colorless oil in 70\% yield. FTIR (neat, $\mathrm{cm}^{-1}$ ) 3409 (b), 2974, 2927 , 2864, 1686, 1115, 1070, 1028; ${ }^{1} \mathrm{H}$ NMR $\left(500 \mathrm{MHz}, \mathrm{CDCl}_{3}\right) \delta$ 7.32-7.24 (m, 10H), 4.19-4.12 (m, $4 \mathrm{H}), 4.04-3.71(\mathrm{~m}, 10 \mathrm{H}), 3.54\left(\mathrm{dd}, J_{l}=8 \mathrm{~Hz}, J_{2}=7 \mathrm{~Hz}, 1 \mathrm{H}\right), 2.84\left(\mathrm{dd}, J_{l}=16 \mathrm{~Hz}, J_{2}=2.5 \mathrm{~Hz}, 1 \mathrm{H}\right)$, 2.73-2.68 (m, 3H), 2.33-2.27 (m, 2H), 2.04-1.84 (m, 2H), 1.58-1.54 (m, 1H), ${ }^{13} \mathrm{C}$ NMR (100 MHz, $\left.\mathrm{CDCl}_{3}\right) \delta 198.8,198.6,137.0,128.8,128.7,127.5,71.2,70.5,70.1,69.8,68.3,68.2,49.1,49.0,44.6$, 44.5, 33.4, 28.9, 28.0; MS ( $\left.\mathrm{APCI}^{+}\right)$calcd for $\mathrm{C}_{14} \mathrm{H}_{19} \mathrm{O}_{3} \mathrm{~S}(\mathrm{M}+\mathrm{H})^{+}:$: 267.1, found 267.0. 
Table 1, Entry 6

3-Hydroxy-hept-6-ynethioic acid S-benzyl ester: Obtained as a colorless oil in 65\% yield. FTIR (neat, $\left.\mathrm{cm}^{-1}\right) 3293,3062,3029,2923,2117,1679,1496,1453,1071,993 ;{ }^{1} \mathrm{H}$ NMR (500 MHz, $\left.\mathrm{CDCl}_{3}\right) \delta$ 7.32-7.24 (m, 5H), 4.25-4.21 (m, $\left.1 \mathrm{H}\right), 4.18-4.12(\mathrm{~m}, 2 \mathrm{H}), 2.83(\mathrm{bs}, 1 \mathrm{H}), 2.79\left(\mathrm{dd}, J_{I}=16\right.$ $\left.\mathrm{Hz}, J_{2}=4 \mathrm{~Hz}, 1 \mathrm{H}\right), 2.74\left(\mathrm{dd}, J_{I}=16 \mathrm{~Hz}, J_{2}=8 \mathrm{~Hz}, 1 \mathrm{H}\right), 2.37-2.33(\mathrm{~m}, 2 \mathrm{H}), 1.98-1.96(\mathrm{~m}, 1 \mathrm{H}), 1.72-$ $1.65(\mathrm{~m}, 2 \mathrm{H}) ;{ }^{13} \mathrm{C}$ NMR $\left(100 \mathrm{MHz}, \mathrm{CDCl}_{3}\right) \delta 198.5,137.1,128.8,128.7,127.4,83.6,69.0,67.3$, 50.2, 34.8, 33.3, 14.7; $\mathrm{MS}\left(\mathrm{APCI}^{+}\right)$calcd for $\mathrm{C}_{14} \mathrm{H}_{17} \mathrm{O}_{2} \mathrm{~S}(\mathrm{M}+\mathrm{H})^{+}:$249.1, found 249.0.

\section{Table 1, Entry 7}

3-Hydroxy-3-phenyl-thiopropionic acid S-benzyl ester: Obtained as a colorless oil in $22 \%$ yield. FTIR (neat, $\left.\mathrm{cm}^{-1}\right) 3454$ (b), 1680, 1495, 1453, 1054, 1027, 979; ${ }^{1} \mathrm{H}$ NMR $\left(500 \mathrm{MHz}, \mathrm{CDCl}_{3}\right) \delta$ 7.36$7.26(\mathrm{~m}, 10 \mathrm{H}), 5.21\left(\mathrm{dd}, J_{I}=9 \mathrm{~Hz}, J_{2}=3.5 \mathrm{~Hz}, 1 \mathrm{H}\right), 4.20-4.15(\mathrm{~m}, 2 \mathrm{H}), 3.03\left(\mathrm{dd}, J_{1}=16 \mathrm{~Hz}, J_{2}=9\right.$ $\mathrm{Hz}, 1 \mathrm{H}), 2.94\left(\mathrm{dd}, J_{I}=16 \mathrm{~Hz}, J_{2}=3.5 \mathrm{~Hz}, 1 \mathrm{H}\right) ;{ }^{13} \mathrm{C} \mathrm{NMR}\left(125 \mathrm{MHz}, \mathrm{CDCl}_{3}\right) \delta 198.4,142.5,137.3$,

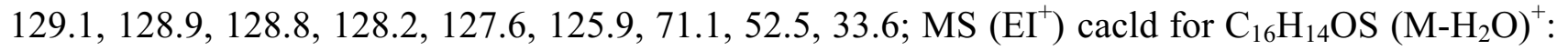
254.0, found 253.8 .

\section{Table 1, Entry 8}

3-Hydroxy-3-(3-nitro-phenyl)-thiopropionic acid S-benzyl ester: Obtained as a colorless oil in $82 \%$ yield. FTIR (neat, $\mathrm{cm}^{-1}$ ) 3508 (b), 3087, 3030, 2926, 1679, 1526, 1348, 1065; ${ }^{1} \mathrm{H}$ NMR (500 $\left.\mathrm{MHz}, \mathrm{CDCl}_{3}\right) \delta 8.27(\mathrm{~s}, 1 \mathrm{H}), 8.16\left(\mathrm{dd}, J_{1}=8 \mathrm{~Hz}, J_{2}=2 \mathrm{~Hz}, 1 \mathrm{H}\right), 7.71(\mathrm{~d}, J=8 \mathrm{~Hz}, 1 \mathrm{H}), 7.53(\mathrm{t}$, $J=8 \mathrm{~Hz}, 1 \mathrm{H}), 7.30-7.27(\mathrm{~m}, 5 \mathrm{H}), 5.32-5.30(\mathrm{~m}, 1 \mathrm{H}), 4.22-4.16(\mathrm{~m}, 2 \mathrm{H}), 3.43(\mathrm{~d}, 3.5 \mathrm{~Hz}, 1 \mathrm{H}(\mathrm{OH}))$, 3.03-3.05 (m, 2H); ${ }^{13} \mathrm{C}$ NMR $\left(100 \mathrm{MHz}, \mathrm{CDCl}_{3}\right) \delta 197.9,148.4,144.2,136.7,131.7,129.6,128.8$, 128.7, 127.5, 122.8, 120.8, 69.8, 51.7, 33.4; $\mathrm{MS}\left(\mathrm{EI}^{+}\right)$calcd for $\mathrm{C}_{16} \mathrm{H}_{15} \mathrm{NO}_{4} \mathrm{~S}(\mathrm{M})^{+}$: 317.1, found 316.7 .

\section{Table 1, Entry 9}

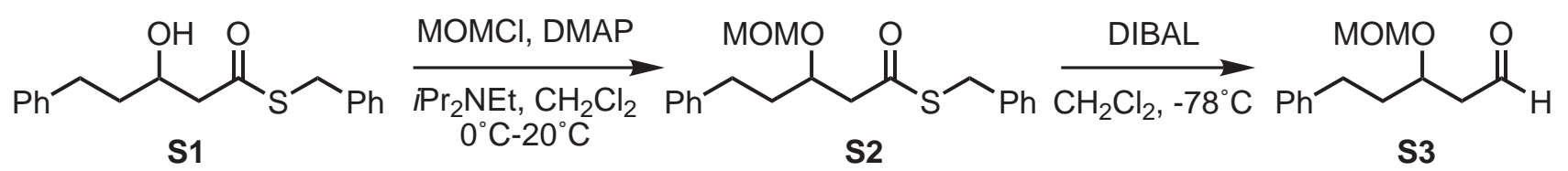

(S2): To a solution of $\mathbf{S 1}\left(105.0 \mathrm{mg}, 0.349 \mathrm{mmol}, 1.0\right.$ equiv) in $\mathrm{CH}_{2} \mathrm{Cl}_{2}(1.5 \mathrm{~mL})$ at $0^{\circ} \mathrm{C}$ was added Hunig's base ( $128 \mu \mathrm{l}, 0.734 \mathrm{mmol}, 2.1$ equiv) followed by methoxymethylchloride ( $53 \mu \mathrm{l}, 0.699$ mmol, 2.0 equiv) and 4-(dimethylamino)pyridine $(8.5 \mathrm{mg}, 0.069 \mathrm{mmol}, 0.2$ equiv). The solution was stirred at $23^{\circ} \mathrm{C}$ for $12 \mathrm{~h}$. The reaction mixture was diluted with EtOAc and washed successively with $0.5 \mathrm{M} \mathrm{HCl}$, saturated aq. $\mathrm{NaHCO}_{3}$, and brine. The organic layer was dried over oven dried $\mathrm{Na}_{2} \mathrm{SO}_{4}$, filtered through cotton, and concentrated under reduced pressure. The product was purified using continuous gradient flash column chromatography (0-15\% EtOAc in hexane) to yield 113.2 $\mathrm{mg}$ of the product $\mathbf{S 2}$ as a colorless oil (94\% yield). FTIR (neat, $\left.\mathrm{cm}^{-1}\right)$ 2930, 1685, 1495, 1454, $1147,1098,1030,917 ;{ }^{1} \mathrm{H}$ NMR $\left(500 \mathrm{MHz}, \mathrm{CDCl}_{3}\right) \delta$ 7.29-7.16 (m, 10H), $4.64(\mathrm{~d}, J=7 \mathrm{~Hz}, 1 \mathrm{H})$, $4.63(\mathrm{~d}, J=7 \mathrm{~Hz}, 1 \mathrm{H}), 4.13(\mathrm{~s}, 2 \mathrm{H}), 4.10-4.06(\mathrm{~m}, 1 \mathrm{H}), 3.35(\mathrm{~s}, 3 \mathrm{H}), 2.92\left(\mathrm{dd}, J_{1}=15 \mathrm{~Hz}, J_{2}=7 \mathrm{~Hz}\right.$, $1 \mathrm{H}), 2.77-2.71(\mathrm{~m}, 2 \mathrm{H}), 2.67-2.61(\mathrm{~m}, 1 \mathrm{H}), 1.91-1.85(\mathrm{~m}, 2 \mathrm{H}),{ }^{13} \mathrm{C}$ NMR $\left(125 \mathrm{MHz}, \mathrm{CDCl}_{3}\right) \delta$ 196.2, 141.5, 137.4, 128.8, 128.5, 128.3, 128.2, 127.2, 125.9, 96.1, 74.4, 55.7, 49.0, 36.5, 33.3, 31.3; MS ( $\mathrm{ES}^{+}$) calcd for $\mathrm{C}_{20} \mathrm{H}_{24} \mathrm{O}_{3} \mathrm{SNa}(\mathrm{M}+\mathrm{Na})^{+}: 367.1$, found 367.3 . 
(S3): To a solution of $\mathbf{S 2}\left(103.4 \mathrm{mg}, 0.309 \mathrm{mmol}, 1.0\right.$ equiv) in $\mathrm{CH}_{2} \mathrm{Cl}_{2}(3.0 \mathrm{~mL})$ at $-78^{\circ} \mathrm{C}$ was added diisobutylaluminum hydride (1 M solution in hexane) $(386 \mathrm{~mL}, 0.386 \mathrm{mmol}, 1.25$ equiv). After 10 min the reaction was quenched with Rochelle's salt and warmed to room temperature. The reaction mixture was diluted with EtOAc and washed successively with Rochelle's salt and brine. The organic layer was dried over oven dried $\mathrm{Na}_{2} \mathrm{SO}_{4}$, filtered through cotton, and concentrated under reduced pressure. The product was purified through a plug of Florisil, using $20 \mathrm{~mL}$ of hexane to separate non polar impurities and $20 \mathrm{~mL}$ of EtOAc to elute product, yielding $72.1 \mathrm{mg}$ of the crude product $\mathbf{S 3}$ as a colorless oil. Product $\mathbf{S 3}$ was used in the next step without further purification.

3-Hydroxy-5-methoxymethoxy-7-phenyl-heptanethioic acid S-benzyl ester: The reaction was performed on a $0.31 \mathrm{mmol}$ scale. The product was btained as a colorless oil in $74 \%$ yield based on the theoretical yield for the previous reaction (see procedure for S3). Diastereoisomers (1.28:1 ratio) were separated by preparative HPLC (Zorbax CN $9.4 \mathrm{~mm}$ column with gradient 5-20\% EtOAc in hexane over $20 \mathrm{~min}$ ).

Major diastereoisomer: FTIR (neat, $\mathrm{cm}^{-1}$ ) 3459 (b), 2938, 1684, 1454, 1147, 1096, 1071, 1028; ${ }^{1} \mathrm{H}$ NMR $\left(500 \mathrm{MHz}, \mathrm{CDCl}_{3}\right) \delta$ 7.29-7.18 (m, 10H), $4.69(\mathrm{~s}, 2 \mathrm{H}), 4.40-4.36(\mathrm{~s}, 1 \mathrm{H}), 4.19-4.12(\mathrm{~m}, 2 \mathrm{H})$, 3.89-3.86 (m, 1H), $3.42(\mathrm{~s}, 3 \mathrm{H}), 2.79\left(\mathrm{dd}, J_{1}=15.5 \mathrm{~Hz}, J_{2}=8.5 \mathrm{~Hz}, 1 \mathrm{H}\right), 2.73-2.63(\mathrm{~m}, 3 \mathrm{H}), 2.17-$ $2.15(\mathrm{~m}, 1 \mathrm{H}), 1.94-1.81(\mathrm{~m}, 2 \mathrm{H}), 1.72-1.64(\mathrm{~m}, 2 \mathrm{H}) ;{ }^{13} \mathrm{C} \mathrm{NMR}\left(100 \mathrm{MHz}, \mathrm{CDCl}_{3}\right) \delta 197.7,141.8$, 137.3, 128.8, 128.6, 128.4, 128.3, 127.3, 125.9, 96.6, 75.4, 65.2, 56.0, 50.8, 41.0, 36.7, 33.3, 31.5; MS (APCI ${ }^{+}$) calcd for $\mathrm{C}_{22} \mathrm{H}_{29} \mathrm{O}_{4} \mathrm{~S}(\mathrm{M}+\mathrm{H})^{+}: 389.2$, found 388.9

Minor diastereoisomer: FTIR (neat, $\mathrm{cm}^{-1}$ ) 3469 (b), 2942, 1684, 1496, 1454, 1147, 1028; ${ }^{1} \mathrm{H}$ NMR $\left(500 \mathrm{MHz}, \mathrm{CDCl}_{3}\right) \delta 7.26-7.14(\mathrm{~m}, 10 \mathrm{H}), 4.67(\mathrm{~d}, J=7 \mathrm{~Hz}, 1 \mathrm{H}), 4.63(\mathrm{~d}, J=7 \mathrm{~Hz}, 1 \mathrm{H}), 4.26-4.21$ $(\mathrm{m}, 1 \mathrm{H}), 4.15-4.09(\mathrm{~m}, 2 \mathrm{H}), 3.82-3.79(\mathrm{~m}, 1 \mathrm{H}), 3.37(\mathrm{~s}, 3 \mathrm{H}), 2,78-2,69(\mathrm{~m}, 2 \mathrm{H}), 2.63-2.60(\mathrm{~m}, 2 \mathrm{H})$, 2.14-2.12 (m, 1H), 1.87-1.76 (m, 3H), 1.68-1.65 (m, 1H); ${ }^{13} \mathrm{C}$ NMR $\left(125 \mathrm{MHz}, \mathrm{CDCl}_{3}\right) \delta 197.8$, $141.8,137.2$, 128.8, 128.6, 128.4, 128.3, 127.3, 125.9, 95.4, 76.3, 67.4, 55.9, 50.7, 40.7, 36.1, 33.3, 31.1; HRMS $\left(\mathrm{ES}^{+}\right)$calcd for $\mathrm{C}_{22} \mathrm{H}_{28} \mathrm{O}_{4} \mathrm{SNa}(\mathrm{M}+\mathrm{Na})^{+}: 411.1606$, found 411.1619.

3-Hydroxy-2-methyl-5-phenyl-pentanethioic acid $\boldsymbol{S}$-benzyl ester (5): This reaction was performed in a capped glass vial that was not treated or dried prior to the experiment. No measures were taken to protect the reaction from the air. To a solution of methyl malonic acid half benzylthioester (3) (25.3 $\mathrm{mg}, 0.113 \mathrm{mmol}, 1$ equiv) in THF (1.1 $\mathrm{mL})$ was added dihydrocinnamaldehyde (30 mg, $0.226 \mathrm{mmol}, 2$ equiv), 5-methoxybenzimidazole (3.3 $\mathrm{mg}, 0.023$ mmol, 0.2 equiv), and $\mathrm{Cu}$ (2-ethylhexanoate $)_{2}(7.9 \mathrm{mg}, 0.023 \mathrm{mmol}, 0.2$ equiv). The green solution was stirred at $23^{\circ} \mathrm{C}$ for $18.5 \mathrm{hr}$, diluted with EtOAc, and washed successively with $0.5 \mathrm{M} \mathrm{HCl}$, saturated aq. $\mathrm{NaHCO}_{3}$, and brine. The organic layer was dried over $\mathrm{MgSO}_{4}$, filtered, and concentrated to a yellow oil. Silica gel chromatography (0 to 18\% EtOAc/hexane continuous gradient) afforded 5 as a yellow oil (18.4 mg, 52\%) and a $7.3: 1$ syn/anti mixture of diastereomers. $\mathrm{R}_{\mathrm{f}}=0.23$ (20\% EtOAc/hexane); FTIR (neat, $\mathrm{cm}^{-1}$ ) 3453, 2927, 1677, 1495, 1454; ${ }^{1} \mathrm{H}$ NMR (500 $\left.\mathrm{MHz}, \mathrm{CDCl}_{3}\right) \delta$ 7.32-7.18 (m, 10H), $4.14(\mathrm{~s}, 2 \mathrm{H}$, minor isomer), 4.13 (s, 2H, major isomer), 3.95 (dt, $J=9.5,4 \mathrm{~Hz}, 1 \mathrm{H}$, major isomer) $3.76-3.70(\mathrm{~m}, 1 \mathrm{H}$, minor isomer), 2.88-2.82 $(\mathrm{m}, 1 \mathrm{H}), 2.74-2.69$ $(\mathrm{m}, 1 \mathrm{H}), 2.68-2.62(\mathrm{~m}, 1 \mathrm{H}), 2.43(\mathrm{br} \mathrm{s}, 1 \mathrm{H}), 1.85-1.77(\mathrm{~m}, 1 \mathrm{H}), 1.71-1.64(\mathrm{~m}, 1 \mathrm{H}) 1.26(\mathrm{~d}, J=7 \mathrm{~Hz}$, $3 \mathrm{H}$, minor isomer) $1.25\left(\mathrm{~d}, J=7 \mathrm{~Hz}, 3 \mathrm{H}\right.$, major isomer); ${ }^{13} \mathrm{C} \mathrm{NMR}\left(100 \mathrm{MHz}, \mathrm{CDCl}_{3}\right) \delta 203.6$, 141.9, 137.4, 129.0, 128.9, 128.7, 127.6, 126.2, 71.6, 53.2, 36.1, 33.4, 32.5, 11.8; HRMS (ES ${ }^{+}$calcd for $\mathrm{C}_{19} \mathrm{H}_{23} \mathrm{O}_{2} \mathrm{~S}(\mathrm{M}+\mathrm{H})^{+}: 315.1419$, found 315.1411 .

3-Benzylsulfanylcarbonyl-2-hydroxy-butyric acid ethyl ester (7): This reaction was performed in a capped glass vial that was not treated or dried prior to the experiment. No measures were taken to 
protect the reaction from the air. To a solution of methyl malonic acid half benzylthioester (3) (112 $\mathrm{mg}, 0.5 \mathrm{mmol}, 1.5$ equiv) in THF $(3.3 \mathrm{~mL})$ was added a $50 \%$ solution of ethyl glyoxalate in toluene (66 $\mu \mathrm{L}, 0.33 \mathrm{mmol}, 1.0$ equiv), 5-methoxybenzimidazole $(9.9 \mathrm{mg}, 0.067 \mathrm{mmol}, 0.2 \mathrm{equiv})$, and $\mathrm{Cu}$ (2-ethylhexanoate) $)_{2}\left(23 \mathrm{mg}, 0.067 \mathrm{mmol}, 0.2\right.$ equiv). The green solution was stirred at $23^{\circ} \mathrm{C}$ for 1 $\mathrm{hr}$, diluted with EtOAc, and washed successively with $0.5 \mathrm{M} \mathrm{HCl}$, saturated aq. $\mathrm{NaHCO}_{3}$, and brine. The organic layer was dried over $\mathrm{MgSO}_{4}$, filtered, and concentrated to a yellow oil. Silica gel chromatography (0 to 30\% EtOAc/hexane continuous gradient) afforded 7 as a yellow oil (76.7 $\mathrm{mg}$, $82 \%)$ and a $7.5: 1$ syn/anti mixture of diastereomers. $\mathrm{R}_{\mathrm{f}}=0.64$ (50\% EtOAc/hexane); FTIR (neat, $\left.\mathrm{cm}^{-1}\right) 3487,2926,1733,1680,1454 ;{ }^{1} \mathrm{H}$ NMR $\left(500 \mathrm{MHz}, \mathrm{CDCl}_{3}\right) \delta 7.32-7.23(\mathrm{~m}, 5 \mathrm{H}), 4.60(\mathrm{~d}, J=$ $3.5 \mathrm{~Hz}, 1 \mathrm{H}), 4.25(\mathrm{q}, J=7.5 \mathrm{~Hz}, 2 \mathrm{H}), 4.16(\mathrm{~d}, J=3.5 \mathrm{~Hz}, 2 \mathrm{H}$, major isomer), $4.13(\mathrm{~d}, J=3 \mathrm{~Hz}, 2 \mathrm{H}$, minor isomer), 3.18-3.13 (m, $1 \mathrm{H}$, minor isomer), 3.11-3.06 (m, $1 \mathrm{H}$, major isomer), $3.02(\mathrm{br} \mathrm{s}, 1 \mathrm{H})$, $1.37\left(\mathrm{~d}, J=7.5,3 \mathrm{H}\right.$, minor isomer), $1.28(\mathrm{t}, J=7 \mathrm{~Hz}, 3 \mathrm{H}), 1.25\left(\mathrm{~d}, J=7 \mathrm{~Hz}, 3 \mathrm{H}\right.$, major isomer); ${ }^{13} \mathrm{C}$ NMR $\left(100 \mathrm{MHz}, \mathrm{CDCl}_{3}\right) \delta 200.1,173.3,137.4,129.1,128.9,127.6,72.9$ (minor isomer), 71.7 (major isomer), 62.5, 51.3, 33.5 (major isomer), 33.4 (minor isomer), 14. 4 (major isomer), 14.0 (minor isomer), 11.6; HRMS $\left(\mathrm{ES}^{+}\right.$) calcd for $\mathrm{C}_{14} \mathrm{H}_{19} \mathrm{O}_{3} \mathrm{~S}(\mathrm{M}+\mathrm{H})^{+}$: 283.1004, found 283.1017.

Determination of diastereoselectivity: Diastereomeric ratios were determined by ${ }^{1} \mathrm{H}$ NMR spectroscopy. Spectral assignment of syn and anti isomers was accomplished by comparison to authentic samples of both the syn and anti diastereomers of 3-hydroxy-2-methyl-5-phenylpentanethioic acid $S$-phenyl ester independently synthesized according to published procedures. ${ }^{2}$ Assignments were in agreement with established trends in the ${ }^{1} \mathrm{H}$ and ${ }^{13} \mathrm{C}$ NMR resonances of aldol adducts. $^{3}$

\section{Crossover experiment:}

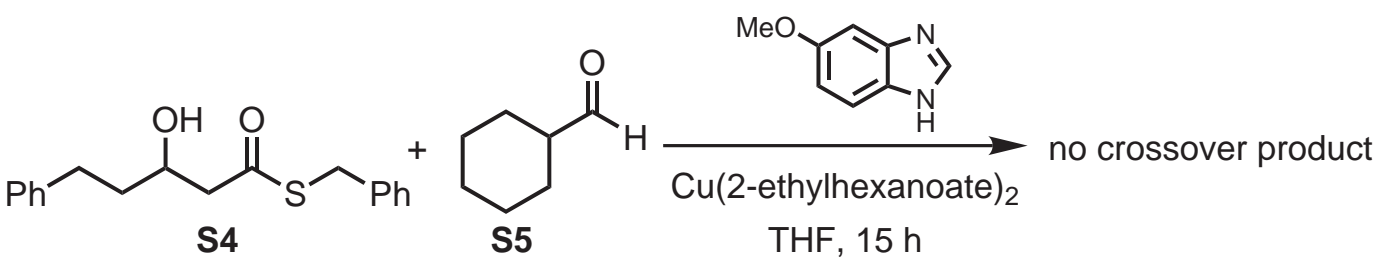

This reaction was performed in a capped glass vial that was not treated or dried prior to the experiment. No measures were taken to protect the reaction from the air. To a solution of S4 (38.2 $\mathrm{mg}, 0.127 \mathrm{mmol}, 1.0$ equiv) in THF $(1.3 \mathrm{~mL})$ was added $\mathbf{S 5}(31 \mu 1,0.254 \mathrm{mmol}, 2.0$ equiv $)$ followed by $\mathrm{Cu}$ (2-ethylhexanoate) $)_{2}(10.1 \mathrm{mg}, 0.025 \mathrm{mmol}, 0.20$ equiv) and 5-methoxybenzimidazole (4.1 $\mathrm{mg}$, $0.028 \mathrm{mmol}, 0.22$ equiv). The solution was stirred at room temperature for $15 \mathrm{~h}$ and quenched with $0.5 \mathrm{M} \mathrm{HCl}$. The resulting solution was diluted with EtOAc and washed successively with $0.5 \mathrm{M}$ $\mathrm{HCl}$, saturated aq. $\mathrm{NaHCO}_{3}$, and brine. The organic layer was dried over oven dried $\mathrm{Na}_{2} \mathrm{SO}_{4}$, filtered through cotton, and concentrated under reduced pressure. The crude ${ }^{1} \mathrm{H}$ NMR revealed no crossover product.

(1) Imamoto, T.; Kodera, M.; Yokoyama, M. Bull. Chem. Soc. Jpn. 1982, 55, 2303.

(2) syn: Hirama, M.; Garvey, D. S.; Lu, L. D.-L.; Masamune, S. Tetrahedron Lett. 1979, 41, 3937. anti: Hirama, M.; Masamune, S. Tetrahedron Lett. 1979, 24, 2225.

(3) Heathcock, C. H. "The Aldol Addition Reaction” in Asymmetric Synth.; Ed. Morrison, James D., 1984, Vol. 3, p. 111. 